\title{
Developing and Evaluating Teaching Material for Information Technology Moral Education for High School Students with Special Needs
}

\author{
Misaki Hashizume, Takeshi Kono, and Shingo Shiota
}

\begin{abstract}
Due to the recent development of information society, use of the Internet is becoming indispensable in assisting people with disabilities to live independent lives. So it is an urgent issue that information technology moral education is provided for children with special needs. However, little research has been conducted on information technology moral education to help such children acquire the necessary communication skills and behaviors.

In this research, educational materials were developed for the information technology moral education of high school students with special needs. The purpose of these teaching materials was to help children learn about the protection of their personal information, and it dealt with content related to the "publication of photographs on SNS."

The results of the student questionnaire survey showed that "I know what photos should not be published on SNS" increased significantly after the lecture compared to before the lecture. In addition, more than $80 \%$ of students responded that "the content of the lecture was easy to understand," suggesting the effectiveness in this material.
\end{abstract}

Index Terms-Special needs education, information technology moral education, SNS communication skills.

\section{INTRODUCTION}

In the past few years, there had been several internet cases reported involving children with special needs. The Japanese Ministry of Education, Culture, Sports, Science and Technology [1] stated that intellectual disabilities could cause people to misread texts, which could result in the person becoming a victim or being involved in a crime without being aware of it. As SNS use by children with intellectual disabilities is increasing, moral information technology education focused on SNS communication skills is needed for people with disabilities at school and at home.

Prior research has reported on social skills training (hereinafter called "SST") at junior and senior high schools to prevent cyberbullying [2] and moral information technology education practices in special needs education [3] However, there has not been any research focused on teaching SNS communication skills to children with special needs.

Manuscript received October 20, 2019; revised March 4, 2020. This work was supported in part by Shizuoka Social Welfare Council Fureai Fund (2018), Fujinokuni Regional / University Consortium Joint Research Grant Program (2019) and Shizuoka University Regional Collaboration Support Project (2019)

The authors are with Shizuoka University, Shizuoka, Japan (e-mail: hashizume.misaki@gmail.com, shiota.shingo@shizuoka.ac.jp).
Therefore, this study developed teaching materials focused on basic SNS behaviors for children with special needs (especially high school students with intellectual disabilities), delivered the materials at a senior high school for special needs education, and evaluated the effect.

\section{METHOD}

To fully understand the moral information technology education situation and the needs of teachers at special support schools, from September to October 2018, a questionnaire was conducted on 84 teachers at four special support schools in Shizuoka, Japan. On the basis of the results of this questionnaire, the teaching materials were developed, and in February 2019, a preliminary session was held for seven third year high school students from a special support school (for children with intellectual disabilities) in Shizuoka, Japan. After the class, an evaluation survey was conducted to assess the viability of the teaching materials.

In July 2019, the modified course was delivered to 17 high school students from the first year to the third year at a special support school. The class was video recorded to allow for a later assessment of the utterances of the students and the students completed pre- and post-questionnaires. A questionnaire was also given to teachers to gain their views on the teaching materials and class content. These data instruments then allowed for a full analysis of the effectiveness of these teaching materials.

All subjects were advised that the questionnaires were only to be used for research, and all the students, school managers, and homeroom teachers gave consent.

\section{PREliminary TEACHER SURVEY}

To reveal the difficulties people with intellectual disabilities have when using information devices and to identify the moral information technology education needed for this student group, a questionnaire was given to 84 teachers working at four special support schools in Shizuoka, Japan.

The first part of the questionnaire asked respondents to give examples of any troubles they had noticed related to the use of information devices by people with intellectual disabilities. It was reported that there had been 65 cases of time wasting, 60 cases of slander, and 54 cases of foolish contributions. For the high school students, respondents reported on 41 cases of encounters through the internet and 
37 cases of personal information leaks. When young, information devices were used to "see" information; however, as the students got older, the internet was used for information "exchange" and therefore, the quality of the types of troubles seemed to change with age.

The next section of the questionnaire asked whether moral information technology education was needed in special support schools. Most (76) respondents felt that such education was very necessary, and six felt that it was needed. The next question asked the respondent teachers whether they had been consulted about the use of the internet and internet games, from which it was found that 38 said that they had been asked by the children, 48 had been consulted by parents, and 20 had never been consulted, which suggested that many teachers were aware of the need for moral information technology education.

Respondents were then asked about the type of instructional content they felt would be appropriate for a high school course from a selection of 13 items. Most felt there needed to be a focus on slander (45), internet encounters (42), personal information protection (36), and social manners (18). As high school provides an education more focused on life after high school, guidance on information technology morals is also necessary to ensure proper behavior in the future.

\section{DEVElopment of THE TEACHING MATERIALS AND PRELIMINARY PRACTICE}

\section{A. Development of Teaching Materials}

On the basis of the results of the preliminary survey, teaching materials for high school students were developed. Generally, people often post photos on SNS. Regarding this, Fujishiro [4] pointed out that analyzing the photos posted by them can reveal the sender's address and workplace. Based on these, it was decided to focus on content related to the exchange of information and in particular, personal information leaks, for which the main theme was photo submissions and communication using photos.

Two main teaching methods were embodied in the teaching material development. First, an SST approach was considered the best method for acquiring social and interpersonal abilities. Sato [5] stated that social skills were learnable, learning could be used to improve and overcome difficulties in interpersonal relationships, and social skills needed to be understood as "specific actions that can be observed" and learned at the action level. When teaching children with intellectual disabilities, it has been found effective to "repeatedly guide them according to actual life situations" [6] so they can make use of the learned content in real life. Therefore, the guidance provided by the SST makes use of an 1) instructions, 2) modeling, 3) role playing, and 4) feedback framework [5] [7]. The teaching material SST flow for the class was as follows.

1) When reviewing troubling scenes, present the skills needed when communicating on SNS and explain in detail the SNS mechanism, merits of practicing the skills, and points to pay attention to. [Instruction and modeling]
2) Deepen student thoughts on information technology morals and skills by reviewing their own thoughts and communicating with others. [Role-playing]

3) Learn how to use the internet and SNS using skills while actually using the equipment or thinking about how to respond to questions from quizzes and teachers. [Role-playing and feedback]

Previous study results on information technology moral education teaching methods were also referred to when developing the materials for paying attention to using SNS and the skills required. For example, Sakai, Shiota, and Eguchi [8] proposed a classification method using cards and comparisons with other people and found that the children were better able to understand the SNS communication problems. Therefore, it was decided that if these types of cards were used to guide children with intellectual disabilities, their opinions could be determined by using the card to visualize the idea, and when comparing with others, the differences in thinking could deepen their understanding.

Using the aforementioned two methods, a teaching materials package was developed that included class slides, cards, and worksheets, the details of which are described in the following.

First, as in (1), a brief explanation of SNS is given and participant usage and troubling experiences discussed. Then, to provide perspective on SNS behavior, a bad example is modeled. A scene is presented in which a person put a picture of a friend's face on SNS without permission, and students are asked to think about why she is surprised by that action.

Next, we explained the structure and overview of SNS. Sugaya [9] stated that it is important to understand the mechanisms of various services in order to protect their own information behavior. Therefore, while exemplifying the applications that the students use most often, such as Twitter, YouTube, and LINE (talk and timeline), the students are asked to think about who might be watching when their photos and videos are being published in each application; the answers to which can be generally divided into two depending on the scope of disclosure: 1) SNS that are accessible to people all over the world (Twitter, Instagram, etc.) and 2) SNS that only allows communication with specific partners (LINE, e-mail, etc.). In addition, it is explained that in the SNS that many people see (1), there is a high possibility that personal information and privacy can be violated by photos.

On the basis of this general explanation, as in (2), a classification and discussion activity is conducted using the card materials. Illustration cards similar to the photographs in SNS posts are presented, and the students are asked to classify the possible posting based on the two SNS types. The names of each item are replaced with words that the students can understand: those that can be posted in 1) are defined as published to the SNS that many people see, and those that can be posted in 2) are defined as published to the SNS that only those who you want to send to can see. If it is considered that posting to both types is dangerous, students choose the answer that it is better not to publish to SNS. Two sets of five cards are prepared; the contents of which are similar: photos that do not include personal information such as sightseeing spots or dishes; photos with personal information such as 
addresses or telephone numbers; selfie photos with friends or photos of acquaintances; photos that show where you are such as at home or school; and naked photos of self (including only the upper body). The first set of cards were developed as the basics and the second set of cards were developed as the applications. The first is used for a thinking activity, and the second set is similar to the first and used for repeated consideration activities.

Finally, as in (3), while looking back at the card classification, the actual SNS use is discussed and the content of the lesson summarized, with the main focus being on the things that the students need to be careful about when posting A hands-on activity is then conducted using the photos learned in the card classification for publishing to the SNS that many people see. In this activity, the students take photos in the classroom, after which the instructor chooses several photos from each student and presents them one by one to the class asking the questions "Would you like to publish this photo?" and "Why do you think you could or could not publish that?" to make the students think about the standards and viewpoints for judging whether to publish or not. Then, while looking at each photograph, the instructor's judgment is explained to everyone to reinforce the criteria.

\section{B. Preliminary Practice Using the Teaching Materials}

In February 2018, a 1 h class was held using the developed teaching materials at a high school course at a special support school (for children with intellectual disabilities) in Shizuoka, Japan. The target students were seven senior high school students, four of whom had communication devices such as mobile phones, smartphones, or tablets. When asked about their troubling experience in using SNS, only two students said that they had had some trouble. Table I shows the teaching plans used in the preliminary practice; the class was developed using the class slide in Fig. 1.

To examine the effectiveness of the teaching materials and the practice exercises, a questionnaire was conducted immediately after the class. The targets were the seven students who took the class.

\begin{tabular}{|c|c|c|}
\hline $\begin{array}{l}\text { Time } \\
(\min )\end{array}$ & Contents & $\begin{array}{l}\text { Positioning in } \\
\text { SST }\end{array}$ \\
\hline 10 & Thinking about troubling cases & \multirow{2}{*}{$\begin{array}{l}\text { Instruction and } \\
\text { modeling }\end{array}$} \\
\hline 10 & Introduction to SNS features & \\
\hline 20 & $\begin{array}{l}\text { Divide five photos into three groups } \\
\text { (repeat twice) }\end{array}$ & $\begin{array}{c}\text { Role-playing } \\
\text { and } \\
\text { feedback } \\
\end{array}$ \\
\hline 5 & Summary & \\
\hline 5 & Take some pictures and question & \\
\hline
\end{tabular}

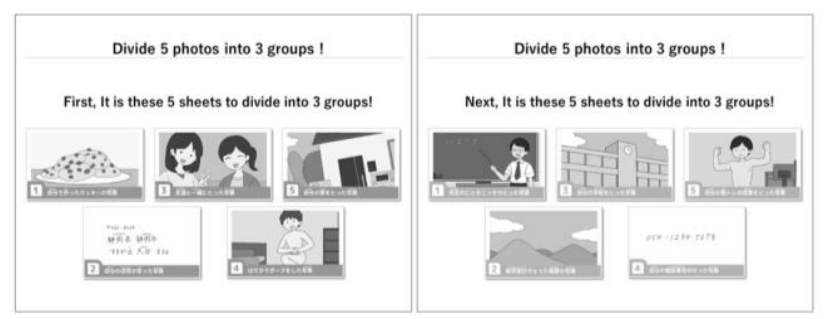

Fig. 1. One of the class slides (teaching materials).
Question 1 asked about the fun of the lesson, and all seven students thought that they had a lot of fun. Question 2 asked whether the students had understood the lesson, with six saying that they had understood it very well and one answering that they had understood it a little. By investigating the students' usage of the devices or applications in advance and giving explanations while showing the names of the most commonly used applications, it was easy for the students to imagine it in real life. Question 3 focused on the practicality of the lesson content, and six students felt that it was very useful.

When asked to write down their impressions, some of the students wrote the following:

- On SNS, I found out what can be published and what should not be published. I don't publish photos on SNS now, but if I do in the future, I will publish it carefully.

- I found out that there are some types of SNS, some photos that I can publish and some photos that I shouldn't publish. I need to think before publishing my photos.

- I have never known the meaning of a timeline until now. But I think I shouldn't use a timeline too much.

In addition to the classifying activity using the card materials, by incorporating actual photo-taking activities, the students appear to have gained an understanding that some things could be disclosed and some should not be disclosed. However, there was no description that a guide as to judging what can be disclosed or what should not be disclosed. Therefore, it was decided that it was necessary to carefully explain the judgment criteria for the classification using the card materials. On the other hand, when there were a lot of detailed information, they were confused to classify, so which seemed to indicate that a careful selection of the amount of information is necessary when instructing.

\section{REVIEW OF THE TEACHING MATERIALS AND THE MAIN PRACTICE}

\section{A. Review of the Materials and an Outline of the Main Practice}

On the basis of the results of preliminary practice, two major revisions were made to the teaching materials and teaching practice.

First, the photography activity, which was performed for a short time after the summary, was put before the summary to be more clearly positioned to the role-playing and feedback. It was surmised from the preliminary practice that even if the student understood the card classification activities, when they took their own photos and needed to make judgment as to whether it was good, they appeared to have some difficulties in judging photos that were different from those on the cards, such as photos of themselves or others and photos of the buildings near the school from the school window. Therefore, we decided to place more emphasis on the positioning of the experience activities. 
Second, the content, amount of presentation information, and presentation method were modified. For example, for the message application LINE, both the talk (one-to-one exchanges and exchanges between groups of specific friends) and timeline (basically, you are registered as a friend) features were explained in the preliminary practice session. However, because the students had compared the timeline feature with the talk feature, it was felt that the explanation regarding the function of the talk feature might have been unclear by adding the timeline feature explanation. Therefore, the timeline feature explanations were deleted and treated as a supplement depending on the actual situation. In addition, regarding the class slides with questions, only the questions were included in the preliminary practice. However, it was thought that the main points could be visualized by clearly specifying the correct answers on each of the slides to make it easier for the students to understand. By describing the correct answers, the correct information could be conveyed to the students even with different instructors; that is, the emphasis was placed on ensuring the materials were easy to understand for the students and easy to teach for the instructors.

The aforementioned modifications were made, and the main practice run at a special support school in July 2019 with 17 high school students from first year to third year, 16 of whom had communication devices such as smartphones and tablets and 7 of whom usually spent more than $2 \mathrm{~h}$ a day on their device.

One student said that he had had a lot of troubling experiences, and one student admitted having a few troubling experiences. The plan for this practice is shown in Table II. The lesson was developed on the basis of the class slide revision from the preliminary practice.

TABLE II: TEACHING PLAN (MAIN PRACTICE)

\begin{tabular}{|c|c|c|}
\hline $\begin{array}{l}\text { Time } \\
(\mathrm{min})\end{array}$ & Contents & $\begin{array}{l}\text { Positioning in } \\
\text { SST }\end{array}$ \\
\hline 5 & Thinking about troubling cases & \multirow{2}{*}{$\begin{array}{l}\text { Instruction and } \\
\text { modeling }\end{array}$} \\
\hline 10 & Introduction to SNS features & \\
\hline 20 & $\begin{array}{l}\text { Divide five photos into three groups } \\
\text { (repeat twice) }\end{array}$ & \multirow{2}{*}{$\begin{array}{c}\text { Role-playing } \\
\text { and } \\
\text { feedback }\end{array}$} \\
\hline 10 & Take some pictures and question & \\
\hline 5 & Summary & \\
\hline
\end{tabular}

\section{B. Analysis of Main Practice Results}

In addition to the pre- and post-student questionnaires, the student utterances were video recorded from the back of the classroom to allow for an analysis of the students' thinking processes and understanding. A teacher questionnaire for the teachers attending the classes was also conducted.

Fig. 2 shows the results from the class questionnaire for 1) class enjoyment; 2) easy-to-understand class; and 3) usefulness of class content. For items 1) and 2), many students felt that the class was fun and easy to understand; however, the few students that answered that it was not fun or they were not sure were students who did not have communication devices or who had them but did not use them daily. It was surmised that the lack of any specific explanation regarding the specific applications and the lack of images of these applications may have led to some students experiencing difficulties with the content.

To measure the change in consciousness before and after the class, students were asked whether they were aware of the differences between photos that may be published online and photos that should not be published online. When the t test was applied to this result, the results shown in Table III were obtained, which indicated that there were significant differences before and after the class and there was a definite effect for increased confidence in being able to distinguish photos that can or cannot be disclosed.

When analyzing the utterances, the following remarks were extracted from the first card activity.

\section{(see photos taken with friends)}

T: Why did you put it here?

$\mathrm{C} 1$ : I'm embarrassed that other people can see the photos. I refuse.

C2: May I send it! Oh, but I don't want other people to see my photos.

C3: If you send this photo, isn't someone going to get various things?

The aforementioned remarks are utterances about how to place a card. Although C3 may be seen from the viewpoint of personal information; that is, there is a risk that various information may be revealed from releasing the photos, it seemed that $\mathrm{C} 1$ and $\mathrm{C} 2$ had decided that it should not be disclosed for their own emotional reasons, such as being embarrassed or not wanting to send the photos.

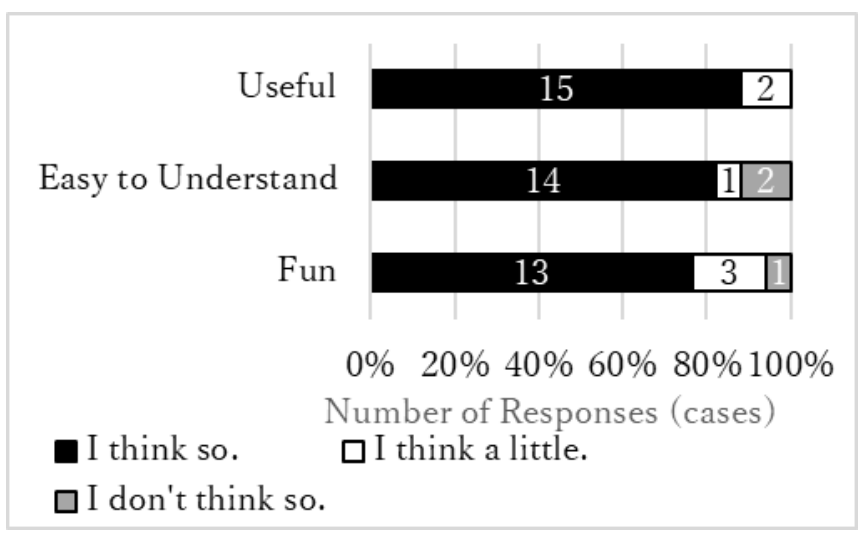

Fig. 2. Post-questionnaire results.

TABLE III: COMPARISON OF AVERAGE VALUES BEFORE AND AFTER THE CLASS

I can see photos that can be published online

\begin{tabular}{|c|c|c|}
\hline Pre & Post & \\
\hline 2.19 & 1.29 & $* *$ \\
$(0.91)$ & $(0.59)$ & \\
\hline \multicolumn{2}{|c|}{$* p<0.01,{ }^{*} p<0.05,()$ is SD }
\end{tabular}

After that, the explanation of the card classification was given again, and the points to be noted were highlighted. The following remarks were given during the second card classification.

(For students who answered that they could share their photos taken during the school trip)

T: Can you tell me why you put it here? Please.

C4: I don't think my personal information will be leaked even 
if I release a picture of the scenery when I went on a school trip.

C1: If it's an SNS that many people see, and everyone often takes trips there, that kind of place is popular and everyone has photos on it.

T: I see. There are lots of other people at popular places, so it's okay to make it public, isn't it?

(Responding to not answering phone numbers)

T: Can you tell me why?

C5: If you put a photo of your phone number on SNS, it will be known to others just like address.

T: Yeah. I see. Then, please give me the reason.

C6: Yes, well, the reason you shouldn't publish is that if you publish, you might be stalked by strangers.

$\mathrm{T}$ : Stalker. That is certainly dangerous.

In this way, based on the first time experience, in the second classification, it seemed that students were able to recognize the risk of publishing photos from a protecting personal information viewpoint, which indicated that the repeated learning had been effective in improving the students' judgment skills as to whether or not to publish photos.

A questionnaire was also conducted on the four teachers attending the classes, with the results being show in Fig. 3 . When the teachers were asked whether they thought the children had deepened their information morals, all said only a little, and in response to the question regarding the usefulness of the course for the children's future work, life, and leisure time, two respondents felt it was very helpful and two respondents felt it was a little helpful, which suggested that the moral information technology teaching had contributed to the children's skills for the limited inline publication of photograph action.

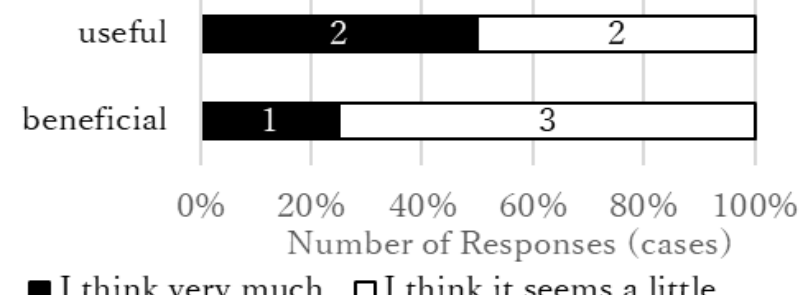

I think very much $\square$ I think it seems a little

Fig. 3. Teacher questionnaire results.

The following are examples of the teachers' impressions.

- I think that it was effective to be able to think and compare them with specific observations and operations on the cards. At a later date, I would like to put the card on the worksheet and file it so that the children can look back.

- It was easy to understand because it specifically explained what kind of photos should be used. The students did not understand, "Why shouldn't I share my friends' photos?", but they understood that personal information shouldn't be published.

As mentioned in the first comment, the classification and comparison became easier because of the use of the card materials as they allowed for a deepening of the students' thoughts on the publishing of certain photos. The second comment related to the publication of friend's photos was not related to personal information but was a copyright issue; however, it was related to the "photo release" skills for an action scene. Therefore, this view should be incorporated in improved teaching materials.

\section{CONCLUSION AND Future ISSUES}

Through this research, it was shown that the usefulness of the developed teaching materials for teaching information technology morals to students with special needs (and especially high school students with intellectual disabilities). After students were trained in what and what not to publish using the card materials, the next session will focus on developing the skills to take photos that may be posted under the premise of the types of things that can be communicated via SNS.

As this study was only run at one special support school, the results need to be verified at other schools. In addition, we also plan to develop teaching materials to verify the effectiveness of SNS communication skills other than the open range photos focused on in this study.

\section{CONFLICT OF INTEREST}

The authors declare no conflict of interest.

\section{AUTHOR CONTRIBUTIONS}

All authors conducted the research; T. Kono and S. Shiota analyzed the data; M. Hashizume wrote the paper; all authors had approved the final version.

\section{REFERENCES}

[1] Ministry of Education, Culture, Sports, Science and Technology, "Information education in special support education," Guide on Computerization of Education, JPN, 2010, pp. 194-222.

[2] Y. Watanabe and E. Harada, "Social skills training for junior high school and high school students: Necessary human relations skills in the smartphone era," JPN, Meiji Books, 2015.

[3] Chieko Nakasuji, "Development and practical verification of teaching materials for information morals at special needs schools," presented at the 43rd Japan Educational Technology Research Council National Convention, Wakayama, JPN, November 24-25, 2017.

[4] H. Fujishiro, Social Media Studies: Redesigning Connections, JPN, Seikyuusya, 2015.

[5] S. Sato, "Social skills education," Practice! Social Skills Education in Elementary School: The Frontline of Classes for Developing Interpersonal Relationship Abilities, JPN, Tosyobunkasha, 2005, pp. 6-15.

[6] Ministry of Education, Culture, Sports, Science and Technology, March 2018 Notification of Special Support School Courses of Study (Department of Elementary and Junior High Schools), JPN, Kairyudo, 2018.

[7] Y. Watanabe "Development of morality and education required for the internet society," Information Moral Education: Internet Communication and Trouble Prevention for Kids to Know, JPN, Kaneko Syobo, 2018, pp. 39-54.

[8] K. Sakai, S. Shiota, and K. Eguchi, "Development and evaluation of a information ethics class for promoting awareness of behavior which leads to problem," Journal of Japan Society for Educational Technology, vol. 39, 2016, pp. 89-92.

[9] K. Sugaya, "Informatics education adapted to changing media environment," Bulletin of the Faculty of Humanities and Social Sciences, Ibaraki University 4, 2019, pp. 61-78. 
Copyright $(92020$ by the authors. This is an open access article distributed under the Creative Commons Attribution License which permits unrestricted use, distribution, and reproduction in any medium, provided the original work is properly cited (CC BY 4.0).

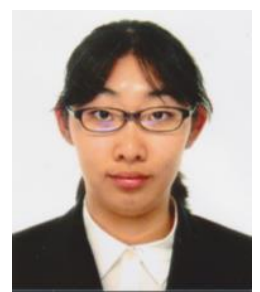

Misaki Hashizume is expected to got the M.A. in education in March 2020, Graduate School of Education, Shizuoka University, Japan. She got the B.A. in education (March 2018) - Faculty of Education, Shizuoka University, Japan.

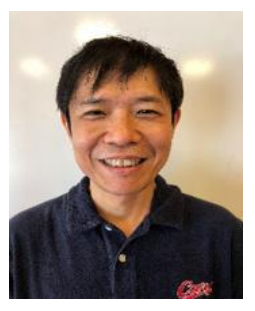

Takeshi Kono is a professor in Faculty of Education, Shizuoka University. He got the Ph.D. in psychology (March 2019) - Graduate School of Human Environment Studies, Kyusyu University, Japan.

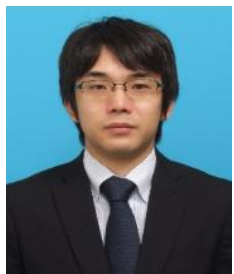

Shingo Shiota is associate professor in Faculty of Education, Shizuoka University. He got the Ph.D. (January 2010) - Graduate School of Environment and Energy Engineering, Waseda University, Japan. 